\title{
Polarization-dependent Imaging Contrast (PIC) Mapping in 2018
}

\section{Pupa U. P. A. Gilbert}

Departments of Physics, Chemistry, Materials Science, Geoscience, University of Wisconsin, Madison WI 53706, USA.pupa@physics.wisc.edu

Ten years after Polarization-dependent Imaging Contrast (PIC) mapping was first introduced $(1,2)$, it has changed and advanced significantly: it now measures the orientation of the crystallographic $c$-axis of crystals in three dimensions (3), and quantitatively displays it in color (4-10).

PIC mapping is based on x-ray linear dichroism, a natural effect occurring in any non-cubic crystal, first discovered by Joachim Stöhr in molecular monolayers (11-13) and later observed in magnetic materials (14-18), organic molecules (19), and liquid crystals (20). All these were human-made systems, whereas Metzler et al. first observed it in a natural sample, nacre from an abalone shell (1). For PIC mapping a PhotoEmission Electron spectroMicroscope (PEEM)(21-26) is used to acquire images at the photon energy position of the most dichroic peaks. Those first observations in nacre $(1,2)$ and sea urchin teeth (27-30) were obtained by the digital ratio of two images, displayed in black and white, and they were not quantitative. See (31) for a comprehensive review. More recently, however, a much more sophisticated and informative method was introduced, in which the angle of the linear polarization from the elliptically polarizing undulator $\left(E P U^{\circ}\right)$ source is rotated by $90^{\circ}$, in $5^{\circ}$ increments. In the resulting stack of 19 images each pixel is fit to a Malus law: $f\left(E P U^{\circ}\right)=\alpha+\beta \cos ^{2}\left(E P U^{\circ}-c^{\prime}\right)$ where $\alpha, \beta$, and $c^{\prime}$ are fit parameters. In the Malus law c' represents the in-plane angle of the crystal's $c$-axis, and $\beta / \alpha$ is the offplane angle. These are displayed as hue and brightness, respectively, in Figure 1. Notice that these inplane and off-plane angles are referred to the polarization plane, which is not the image plane. Since the beam illuminates the sample surface from the right at $30^{\circ}$ grazing incidence, the polarization plane is $60^{\circ}$ from the surface.

Hue in PIC maps displays the $c^{\prime}$ angle, that is, the angle between the projection of the crystal $c$-axis onto the polarization plane, termed $c^{\prime}$-axis, and the vertical in the laboratory and in the images. When the $c^{\prime}-$ axis is vertical, the angle is $0^{\circ}$ and the color is cyan, when the $c^{\prime}$-axis is $-30^{\circ}$ (left from the vertical) the color is blue, at $+30^{\circ}$ is green, and so on as shown in the color legend in Figure 1.

Brightness in PIC maps displays the off-plane angle of the crystal $c$-axis: bright colors indicate in-plane $c$-axes, darker colors are off-plane, and black is $90^{\circ}$ off-plane, that is, the $c$-axis points directly into the $\mathrm{x}$-ray beam (4).

All PIC maps presented here use the default parameter values in the software used to produce them: angle minimum $=-90$, maximum +90 , scale bar colors $0^{\circ}-360^{\circ}$, brightness set to $\max \mathrm{B}=200$. The software is called GG Macros and it is distributed free of charge to any interested users (32).

Figure 1 shows PIC maps from aragonite, calcite, and vaterite in space-filling (30) marine biominerals. These are three polymorphs of calcium carbonate $\left(\mathrm{CaCO}_{3}\right)$ with orthorhombic, trigonal-rhombohedral, and hexagonal crystal structures, respectively. The PEEM resolution in Figure 1 is $20 \mathrm{~nm}$, and the pixel sizes vary between $10 \mathrm{~nm}$ and $60 \mathrm{~nm}$. All these PIC maps were obtained at the oxygen K-edge in PEEM. 


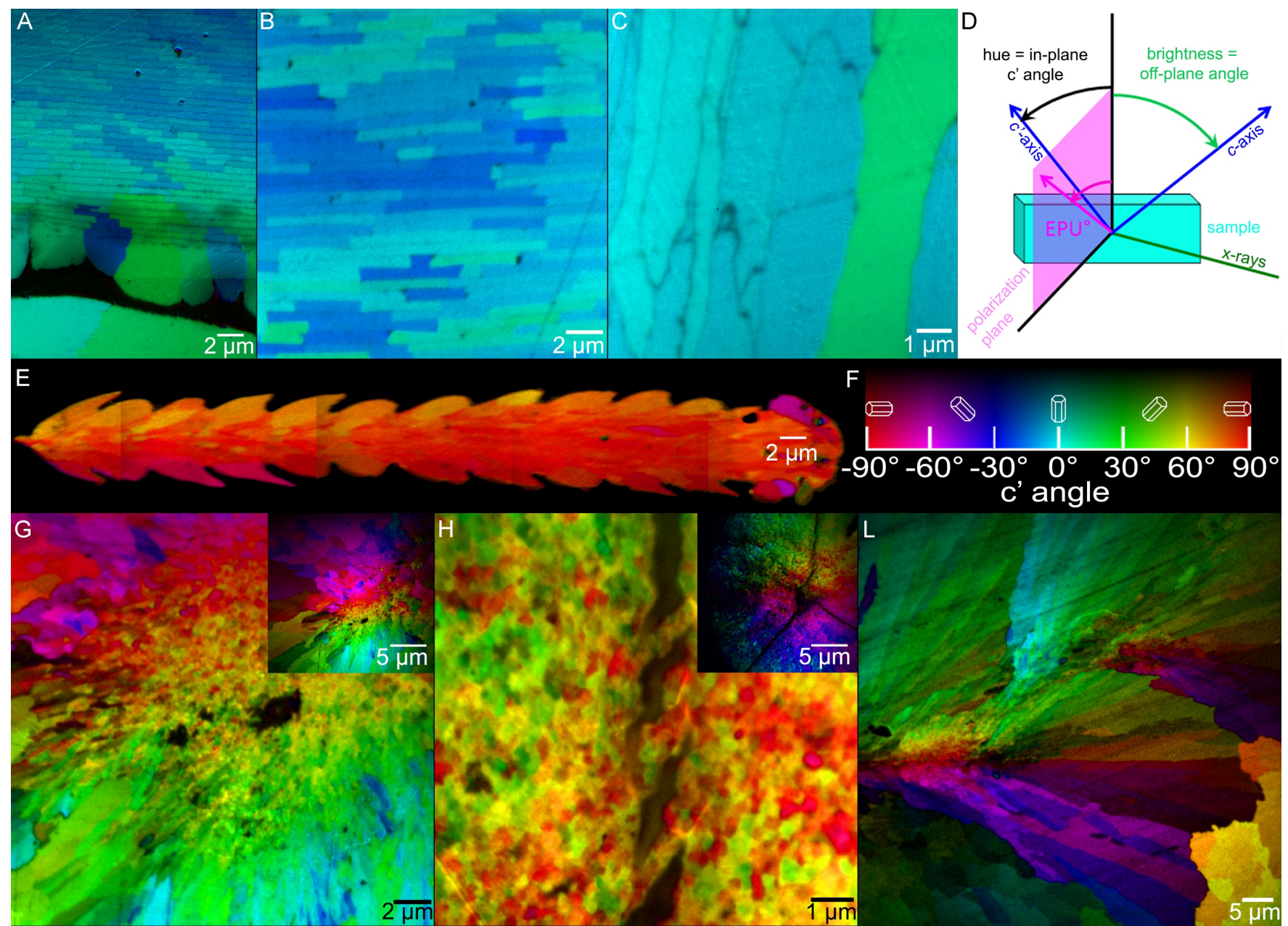

Figure. 1. Polarization-dependent Imaging Contrast (PIC) maps, in which color indicates the carbonate crystal $c$-axis orientation. Pixels sizes are 10,20, or $60 \mathrm{~nm}$ where the scale bars are $1 \mu \mathrm{m}, 2 \mu \mathrm{m}$, or 5 $\mu \mathrm{m}$, respectively. A. Aragonite $\left(\mathrm{CaCO}_{3}\right)$ nacre in Pinctada fucata shell, re-processed in color from (33), demonstrating that near-epitaxial growth (33) and gradual ordering (27) in nacre. B. Nacre in 13 million-year-old Pinna sp. shell, adapted from (7), demonstrating that nacre does not change significantly in fossils. C. Calcite $\left(\mathrm{CaCO}_{3}\right)$ prisms in Mytilus californianus adapted from (4), demonstrating that prisms are not all co-oriented. D. Schematic of the PIC mapping experiment, with the crystal $c$-axis, and its projection $c$ '-axis onto the polarization plane. E. Vaterite $\left(\mathrm{CaCO}_{3}\right)$ spicule from Herdmania momus tunicate (6), showing the largest vaterite crystals ever observed. F. Color bar in which the color's hue and brightness represent the in- and off-plane angles of the crystal $c$-axis, with respect to the polarization plane, which is $60^{\circ}$ from the image plane (see D for geometry). The same color bar applies to all PIC maps here, from all $\mathrm{CaCO}_{3}$ polymorphs. G. Aragonite coral skeleton around centers of calcification (black dots) demonstrating that Stylophora pistillata coral skeletons form by attachment of particles. Inset shows the same region at lower magnification. Data from (9). $\mathbf{H}$. Aragonite spherulite grown in vitro in the absence of any organic additives, demonstrating that synthetic aragonite also forms by attachment of particles. Notice the vertical crack across the entire image, exhibiting crack bridging, another clear indication of particulate material. Inset shows lower magnification PIC map of the same region. Data from (9). L. Another coral skeleton showing a radial distribution of acicular crystals, in which the angular distance of $c$-axes in adjacent crystals is within $30^{\circ}$, demonstrating spherulitic growth of coral skeletons. Data from (10). 
The discoveries enabled by PIC mapping in these marine biominerals are summarized in Figure 1 caption (34). PIC mapping also works for apatite crystals, which are the main constituents of teeth and bone, as recently demonstrated in parrotfish teeth (8). Thus, many new discoveries are forthcoming on the nanoand micro-scale structure of bone and teeth from humans and other animals.

References:

1. RA Metzler et al Phys. Rev. Lett. 98 (2007).

2. RA Metzler et al Phys Rev B 77 (2008), 064110-1.

3. PUPA Gilbert et al Proc Natl Acad Sci USA 108 (2011).

4. RT DeVol et al J Phys Chem B 118 (2014).

5. RT DeVol et al J Am Chem Soc 137 (2015).

6. B Pokroy et al Chem Mater 27 (2015).

7. PUPA Gilbert et al Earth and Planetary Science Letters 460 (2017).

8. MA Marcus et al ACS Nano 11 (2017).

9. T Mass et al Procs Natl Acad Sci 114 (2017).

10. C-Y Sun et al ACS Nano 11 (2017).

11. J Stohr et al Phys. Rev. Lett. 47 (1981).

12. RJ Madix et al Surf. Sci. 197 (1988).

13. J Stohr in NEXAFS Spectroscopy (Springer-Verlag, Berlin)

14. G van der Laan et al Phys Rev B 43 (1991).

15. J Stohr et al Phys. Rev. Lett. 83 (1999).

16. A Scholl et al Science 287 (2000).

17. J Lüning et al Phys Rev B 67 (2003).

18. MB Holcomb et al Phys Rev B 81 (2010).

19. H Ade et al Science 262 (1993).

20. J Stohr et al Science 292 (2001).

21. G De Stasio et al Rev Sci Instrum 70 (1999).

22. B Gilbert et al Ultramicroscopy 83 (2000).

23. G De Stasio et al Ultramicroscopy 98 (2003).

24. M Najman et al Tribol Lett 17 (2004).

25. G De Stasio et al Clin Cancer Res 12 (2006).

26. G Pereira et al Tribol 1 (2007).

27. PUPA Gilbert et al J Am Chem Soc 130 (2008), 17519.

28. CE Killian et al J Am Chem Soc 131 (2009).

29. YR Ma et al Procs Natl Acad Sci USA 106 (2009).

30. L Yang et al RSC-Nanoscale 3 (2011).

31. PUPA Gilbert J Electr Spectrosc Rel Phenom, special issue on Photoelectron microscopy, Timeresolved pump-probe PES 185 (2012).

32. GG-Macros http://home.physics.wisc.edu/gilbert/software.htm (2018).

33. IC Olson et al J Struct Biol 184 (2013).

34. Graduate students Brad H Frazer, Rebecca A Metzler, Ian C Olson, Ross T DeVol, and Chang-Yu Sun's tireless work made biomineral PIC mapping possible. Beamline scientists Andreas Scholl and Anthony Young at ALS provided expert technical support. Susan N Coppersmith and Matthew A Marcus gave theoretical contributions. NSF awards DMR-1105167 and DMR-1603192, and DOE Award DE-FG02-07ER15899 supported this work. Experiments were done at the Berkeley Advanced Light Source, supported by DOE under contract DE-AC02-05CH11231. 
https://doi.org/10.1017/S1431927618014514 Published online by Cambridge University Press 